\author{
Margarida Sotto Mayor ${ }^{1}$ \\ Helena Pestana ${ }^{2}$ \\ Gorete Reis ${ }^{3}$ \\ Constância Carneiro ${ }^{4}$ \\ Marcos Valente ${ }^{5}$
}

1. Doctora en Ciencias Biomédicas. Enfermera especialista en Salud Mental y Psiquiatría. Hospital Magalhães Lemos. UNIFAI. Porto. Portugal.

2. Doctora en Métodos Cuantitativos en Gestión. Profesor auxiliar. Instituto Superior das Ciências do Trabalho e Emprego. UNIFAI. Lisboa. Portugal.

3. Doctora en Enfermería. Profesor coordinador. Universidade de Évora. Escola Superior de Enfermagem de S. João de Deus. UNIFAI. Évora. Portugal.

4. Licenciada. Enfermera. Centre Hospitalier Regional d'Orleans. New Orleans. Francia.

5. Estudiante. Instituto Superior do Serviço Social. Porto. Portugal.

\section{Dolor y demencia en las personas que viven en una unidad de larga estancia}

\author{
Pain and dementia of people \\ living in a nursing home
}

Correspondencia:

Gorete Reis

greis@uevora.pt

\section{RESUMEN}

El dolor es prevalente, infradiagnosticado e infratratado en las personas con diagnóstico de demencia u otros trastornos mentales.

Objetivo: identificar la prevalencia del dolor y su relación con las variables sociodemográficas, clínicas y funcionales de las 80 personas residentes en una unidad de larga estancia (ULE) del centro de Francia.

Métodos: elaboración de un guion orientador para la recogida de datos de variables sociodemográficas, clínicas y funcionales. Registro de dolor.

Resultados: El dolor tiene una prevalencia del $70 \%$ en los residentes de la ULE.

Conclusión: población envejecida, sobre todo del sexo femenino, polimedicada, con pluripatología, dolor y menor conciencia de sí misma. Son necesarias medidas formativas que ayuden a los cuidadores formales e informales a identificar el dolor en las personas que no lo verbalizan y a comprender las alteraciones de comportamiento resultantes del dolor en las personas con deterioro cognitivo.

PALABRAS CLAVE: demencia, dolor, unidad de larga estancia.

\section{ABSTRACT}

Pain is prevalent, sub diagnosed and sub treated in people with dementia or other mental disorders.

Aim: To identify the prevalence of pain and its relation with sociodemographic, clinical and functional variables of 80 residents in a unit of long stay (LSU) in the centre of France.

Methods: Elaboration of an orientation script for data collection of sociodemographic, clinical and functional variables. Register of pain.

Results: The prevalence of pain is $70 \%$ of the residents in a LSU. Conclusion: It's an aged population, mainly women, multi pharmacology and with multi diseases, pain and minor awareness of themselves. It's necessary formative procedure to help formal and informal caregivers to identify pain in those who do not verbalize it and in order to understand deviate behaviour caused by pain in those with cognitive deterioration.

KEYWORDS: dementia, pain, long standing unit.

\section{- INTRODUCCIÓN}

El dolor presenta una elevada prevalencia en las personas que padecen demencia. Algunos estudios han demostrado que la prevalencia del dolor en la comunidad es de cerca del $50 \%$, y en las residencias de ancianos puede alcanzar hasta el $80 \%^{1-4}$. Las estimaciones oscilan entre el $45 \%$ y el 80\%, dependiendo de las características específicas de los enfermos o de los instrumentos de medición utilizados 5 .

En un estudio internacional realizado por Achterberg $(2013)^{5}$ en una unidad de larga estancia (ULE) se verificó que la prevalencia del dolor -definido como cualquier tipo de dolor- oscilaba entre el 32\% en Italia, el 43\% en Holanda y el 57\% en Finlandia. En casi el 50\% de los casos el dolor estaba presente diariamente, y mediante un análisis multivariado se concluyó que el dolor estaba positivamente asociado a una deficiencia física más grave, a depresión clínica y al diagnóstico de osteoporosis, y negativamente relacionado con el diagnóstico de demencia y con las situa- ciones más graves de deterioro cognitivo 5 . Se concluyó que el dolor está presente con frecuencia en las ULE en Europa y que a pesar de las diferencias culturales y de la multitud de casos, el dolor solo habla una lengua ${ }^{1}$.

Durante el transcurso normal de la enfermedad y en la secuencia de la disfunción cognitiva aparecen evidentes síntomas neuropsiquiátricos. Estos síntomas incluyen delirios/alucinaciones, agitación/agresión, disforia/depresión, ansiedad, apatía/indiferencia, desinhibición, irritabilidad/labilidad, comportamiento motor anormal y alteración en el patrón del sueño y del apetito 5 . A lo largo de la vida, cerca del $80-85 \%$ presentan uno o más de estos síntomas neuropsiquiátricos ${ }^{6-8}$.

Existen procesos neuronales especializados que actúan simultáneamente, teniendo como base los tres aspectos psicológicos del dolor: sensación-discriminación, afectividad-motivación y cognición-valoración?. En los procesos demenciales, estos aspectos están alterados, es decir, las personas tienen dificultad para expresarse e interpretar lo que les sucede cuando tienen dolor. La demencia y el dolor son dos fenómenos complejos, considerando 
los diferentes subtipos de demencia y las diversas formas en las que el dolor puede manifestarse (dolor nociceptivo, neuropático y central) ${ }^{10}$. Tal y como sucede con el dolor, el sufrimiento es subjetivo, es algo personal, y solo la propia persona conoce su dimensión real ${ }^{9}$. La subjetividad relativa al dolor provoca que sea extremamente difícil (...) es una experiencia sensorial y emocional desagradable asociada a una lesión tisular concreta o potencial, o que es descrita como tal, por lo que puede existir dolor sin la presencia objetiva de lesión o estímulo 9 . En varios estudios se ha demostrado que existe una gran variabilidad interindividual en la intensidad del dolor que se atribuye a un mismo estímulo nociceptivo. La valoración cognitiva de la información nociceptiva aferente, al colocarla en un contexto personal (experiencias pasadas, situación presente e implicaciones futuras) bajo influencia de factores genéticos y psicológicos, contribuye a transformar la información sensitiva en una experiencia única y subjetiva del dolor ${ }^{11}$. El modo en que el anciano interpreta el dolor puede interferir en la forma de expresar dicho dolor ${ }^{10}$. En este contexto, la verbalización de gemidos, suspiros, gritos, coprolalia o expresiones faciales (arrugar el ceño, muecas) o también las expresiones corporales de inquietud o aumento de tensión (rigidez, balanceado), agitación/agresividad o resistencia a los cuidados son con frecuencia la única característica de dolor ${ }^{12} \mathrm{y}$ no pueden pasar desapercibidos a los profesionales de la salud ni a los familiares/cuidadores ${ }^{13}$. Los profesionales de enfermería desempeñan un papel importante al identificar y valorar el dolor en estas personas ${ }^{14}$. A menudo estos comportamientos son interpretados como síntomas de demencia y, por consiguiente, el dolor está infratratado e infravalorado. Asimismo, en publicaciones científicas figura que el dolor es infravalorado frecuentemente, tanto en los aspectos farmacológicos como en las medidas de confort ${ }^{15,16}$, lo que provoca un gran impacto en la calidad de vida de la persona enferma, así como en la de sus cuidadores al ver su sufrimiento. Las personas con demencia reciben menos analgesia en comparación con las personas sin déficit cognitivo, incluso ante la misma situación médica ${ }^{14}$. Generalmente, cuanto más grave es el estado demencial, mayor dificultad tiene la persona para expresar lo que siente y demostrar su desaliento ${ }^{17}$.

Es necesario realizar una valoración clínica global y minuciosa y los profesionales de enfermería deben observar constantemente las múltiples facetas en las que el dolor se presenta ${ }^{18}$. Estudios recientes indican que "el no reconocimiento del dolor" funciona como una barrera para los cuidadores y simultáneamente provoca que las personas enfermas reciban más psicotrópicos de lo que sería necesario, lo que las hace más vulnerables a los efectos secundarios de estos medicamentos (como por ejemplo mayor riesgo del dolor, somnolencia, estado de ánimo deprimido). Se conoce poco sobre la relación entre el dolor y los episodios de alteración del comportamiento (comportamiento desafiador ${ }^{8,19}$ ), aunque varios estudios han demostrado su correlación ${ }^{20}$. Aliviar el dolor ha sido uno de los grandes objetivos de los profesionales de la salud'. Con este trabajo se pretende dar a conocer los resultados de una investigación realizada en una ULE en el centro de Francia. El objetivo de este estudio ha sido identificar la relación entre los episodios de dolor y las variables sociodemográficas, clínicas y funcionales de las 80 personas residentes en una ULE del centro de Francia.

\section{- MÉTODO}

\section{Participantes}

Participan un total de 80 ancianos con trastorno mental (universo/ muestra), residentes en una ULE de un hospital del centro de Francia. Se trata de un estudio transversal, descriptivo y correlacional.

\section{Instrumento para la recogida de datos}

Escala Aggir $^{21}$ para la dependencia/independencia. Los grados de dependencia de la escala Aggir van del 1 al 6, donde 6 es el nivel más bajo para la independencia; valoración del estado mental (atención, coherencia; comportamiento adecuado, orientación espacial y temporal); presencia de enfermedad; número de medicamentos; aparición de dolor e implementación de medidas de seguridad.

La variable en estudio es la prevalencia del dolor en los ancianos con demencia (incluyendo otros trastornos mentales con deterioro cognitivo), comprobada a través de la evidencia de dolor y de su registro en el proceso clínico.

\section{Procedimientos}

Los datos fueron recogidos por el personal de enfermería previamente instruido. El estudio tuvo lugar en el año 2013 y no se alteraron las rutinas diarias de la prestación de cuidados. Así mismo, se respetaron los principios éticos que orientan la investigación con personas.
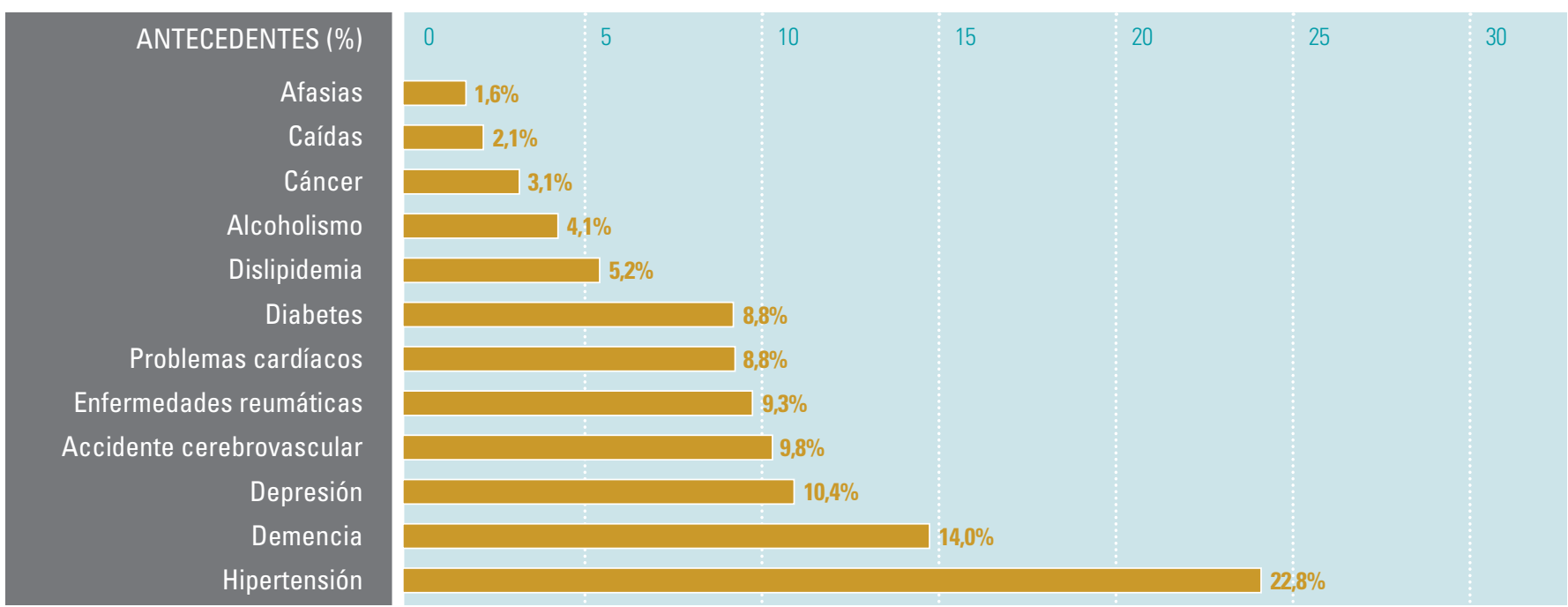

Figura 1. Condiciones patológicas previas. 
El tratamiento estadístico es descriptivo y correlacional con modelos de análisis categórico ${ }^{22,23}$.

\section{- RESULTADOS}

Se trata de una población envejecida donde la mayoría son mujeres (76,3\%) y viudas (62,5\%). La media de edad es de 83,05 ańos y el $50 \%$ de las personas tiene entre 76 y 90,5 años. A pesar de que el valor predictivo del dolor en las personas con más de 80 años sea del $62,5 \%$ y en las personas con menos de 79 años del $37,5 \%$, las diferencias observadas no son estadísticamente significativas. Existe una débil relación positiva entre el aumento de la edad y del dolor, según el coeficiente de correlación biserial que es igual a 0,1158 . Solo el 1,3\% del dolor es atribuible a la edad.

Aunque la mayoría de las personas son viudas, no existen diferencias estadísticamente significativas con relación al estado civil, sexo y dolor, según el test de independencia condicional de Mantel-Haenszel, siendo $p=0,503$. Las personas con dolor son mayoritariamente mujeres, independientemente de su estado civil, aunque las diferencias no son significativas, según el test de $\chi^{2}$ de homogeneidad de las odds ratio de Breslow-Day, siendo sig $=0,306$.

La prevalencia del dolor en la muestra es del 70\%, lo que difiere significativamente de aquellos que no tienen dolor, según el test binomial $(p<0,001)$. El valor predictivo del dolor en las mujeres es del $73,8 \%$, mientras que en los hombres es del 57,9\%. De esta forma, el riesgo de dolor es un $27 \%$ mayor en las mujeres que en los hombres, aunque las diferencias no sean estadísticamente significativas, según el test de $\chi^{2}$ de Fisher, donde sig $=0,252$.

La mayoría de las profesiones representadas son las del grupo de ama de casa y las menos representadas son las del grupo de agricultores. El dolor predomina en las amas de casa, y se presenta conforme a las proporciones observadas en las profesiones, seguida de los obreros y en menor proporción de los agricultores. No obstante, el dolor se sitúa ligeramente por encima de lo esperado en 3,1 de las personas (14-11,9) que tuvieron como actividad dominante los servicios, según la medida de asociación direccional de Goodman y Kruskal, siendo tau $=0,04$ y sig $=0,043$.

La gran mayoría de los antecedentes patológicos personales recae sobre la hipertensión $(22,8 \%)$, seguida de la demencia (14\%), y en menor proporción figuran las afasias y el dolor. Cuando se analizan los antecedentes patológicos personales se verifican dos comportamientos con referencia a episodios de dolor (modelo de regresión logística binaria, test Hosmer-and Lemeshow; sig = 0,261): por un lado, las personas que no indicaron episodios de dolor sufren accidente vascular cerebral (AVC), demencia, depresión, dislipidemia o hipertensión, aunque las diferencias no sean estadísticamente significativas; por otro lado, las personas que indicaron episodios de dolor tienen antecedentes de afasias, alcoholismo, cáncer, enfermedades reumáticas, problemas cardíacos, dolores y diabetes, aunque solo esta última es estadísticamente significativa.

Únicamente las personas con antecedentes de diabetes tienen 5,225 veces más probabilidad de manifestar dolor $(p=0,06)$ que el resto.

La media de medicamentos consumidos se sitúa entre 5,25 (dolor) y 11,41 (diabetes), con diferencias estadísticamente significativas en dos sentidos: menor consumo de medicamentos en las personas con antecedentes de alcoholismo, demencia y dolor, y mayor consumo en los que presentan antecedentes de diabetes y depresión.

\section{- RELACIÓN ENTRE LOS ANTECEDENTES PATOLÓGICOS PERSONALES}

Los que tienen antecedentes de hipertensión (44) comparten igualmente, por orden decreciente de importancia, el diagnóstico de demencia (17), depresión (13), AVC (12), problemas cardíacos (12), enfermedades reumáticas (11) y diabetes (11).

La hipertensión comparte en menor proporción los antecedentes de dolor, afasias, alcoholismo, cáncer y dislipidemia.

Según puede observarse en el gráfico Category Web (fig. 2), la distribución de los antecedentes patológicos relacionados con el dolor tiene lugar con mayor intensidad en las líneas por orden decreciente de grosor.

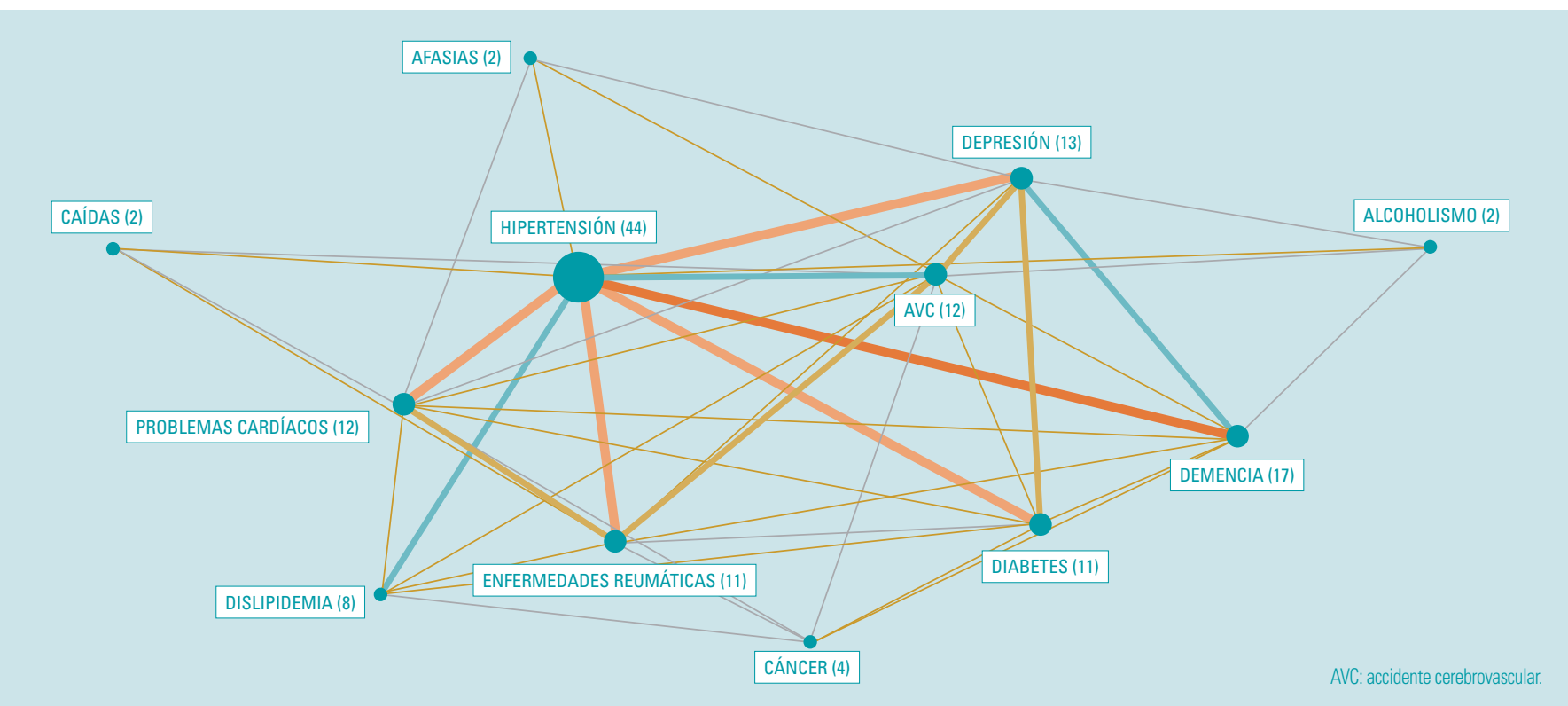

Figura 2. Grado de asociación entre los antecedentes patológicos y el dolor. 
En el análisis del estado mental (atención, coherencia del discurso, adecuación del comportamiento, orientación espacial y temporal) se constata que la mayoría, el 47,5\%, presenta atención captable y que la atención varía en función de los episodios de dolor. Sin embargo, se observa que las personas que tienen atención captable "a veces" y las personas que tienen atención "preservada" presentan respectivamente 2,4 y 2,2 veces más dolor que las personas cuya atención no se consigue captar. No obstante, estas diferencias no son estadísticamente significativas.

La mayoría de las personas no tiene un discurso coherente $(47,5 \%)$, aunque se observa que las que tienen coherencia en el discurso "a veces" y las que tienen siempre coherencia en el discurso presentan respetivamente 1,5 y 1,4 veces más dolor que las que no la tienen; sin embargo, las diferencias no son significativas.

La mayoría de las personas no presenta un comportamiento adecuado, aunque se observa que las personas que tienen comportamiento adecuado "a veces" y las que tienen comportamiento adecuado "siempre" presentan, respetivamente, 1,8 y 1,2 veces más episodios de dolor que las que tienen un comportamiento "inadecuado". Sin embargo, no se diferencian significativamente entre ellas.

Con respecto a la orientación espacial, la mayoría (52\%) no posee orientación, aunque se observa que las que tienen "siempre" orientación espacial sufren 1,6 veces más episodios de dolor que las que no tienen orientación, aunque las diferencias no son significativas.

Lo mismo sucede con la variable orientación temporal, en la que la mayoría $(53,8 \%)$ no posee dicha orientación, aunque se observa que los que tienen orientación "a veces" y "siempre" presentan respectivamente 1,8 y 1,6 veces más episodios de dolor que las que no poseen orientación temporal, aunque las diferencias no son estadísticamente significativas.

Por lo tanto, por lo que respecta al estado mental, se observa que la orientación preservada, la coherencia del discurso y el comportamiento adecuado en cuanto a la orientación espacial y temporal están más asociados a los episodios de dolor que los opuestos.

En cuanto a las enfermedades psicológicas, psiquiátricas y neurológicas que estas personas padecen, se comprueba que la mayoría no posee ninguna enfermedad psicológica ni psiquiátrica $(82,5 \%)$, aunque en los registros clínicos figura una incidencia del 17,5\%; sin embargo, son valores insignificantes para el dolor. Existen otras enfermedades, aunque con baja prevalencia, entre las que destacan las cardiovasculares y las metabólicas; no obstante, únicamente la diabetes presenta diferencias significativas con relación al dolor $(p<0,001)$.

En lo referente a la funcionalidad, las ocho variables que componen la escala Aggir pueden resumirse claramente en un único índice que explica el 71,6\% de la información de dichas variables. Y las diferencias encontradas no varían significativamente con el dolor.

Respecto a los utensilios, se constata que la mayoría (80\%) no se sirve los alimentos de la bandeja, y presentan un riesgo de no manifestar dolor 1,061 veces superior al de los que son capaces de hacerlo, independientemente del sexo. Lo mismo sucede con las variables relacionadas con ordenar la casa, ir a la compra, gestionar el dinero, utilizar transportes y con las medidas de seguridad en la silla o cama, con un riesgo semejante de no manifestar dolor, sin presentar diferencias significativas entre ellas.

\section{Discusión}

Este trabajo presenta una población institucionalizada envejecida, predominantemente de sexo femenino y con dolor. La mayor longevidad induce a la morbilidad, coexistiendo pluripatología en los residentes. El dolor es uno de los síntomas descritos con mayor frecuencia en ancianos con demencia, con tasas de prevalencia que varían del 12\% al $83 \%$. La prevalencia del dolor en esta investigación es del $70 \%$, cifra que coincide con las de otros estudios en los que figuran valores entre el $45 \%$ y el $80 \%{ }^{1-4}$. Las diferencias de género en relación con las manifestaciones de dolor coinciden con las de otros autores ${ }^{24}$. El dolor está asociado a trastornos del humor, y muchas veces da lugar a depresión y/o ansiedad ${ }^{25}$.

Los residentes prescriben medicamentos diariamente y no se utilizan alternativas a los fármacos en esta unidad ${ }^{26}$. La mayoría de las antiguas profesiones representadas no presentan ninguna asociación con episodios de dolor, lo que coincide con otros autores que tampoco identifican ninguna asociación entre las profesiones y el dolor ${ }^{24}$.

Está presente la pluripatología y la fragilidad, al igual que en otros estudios $^{13}$. Verificamos en esta investigación que los que presentan antecedentes de hipertensión comparten, igualmente, por orden decreciente de importancia, el diagnóstico de demencia, depresión, ACV, problemas cardíacos, enfermedades reumáticas y diabetes. Según la mayoría de los autores $^{27}$, la hipertensión sufre un aumento de prevalencia con la edad y está relacionada con otras comorbilidades, concretamente con algunos cambios estructurales y con una mayor rigidez y envejecimiento de órganos y sistemas ${ }^{27}$, lo que coincide con los datos encontrados en la ULE. Únicamente las personas con antecedentes de diabetes presentan 5,225 veces mayor probabilidad de manifestar dolor que el resto. Este dolor es conocido como dolor neuropático, y consiste en un dolor complejo y multifocal grave que aumenta a lo largo del tiempo ${ }^{10}$. Las personas institucionalizadas están frecuentemente polimedicadas ${ }^{13}$. En este estudio, la media de medicamentos consumidos se sitúa entre 5,25 (dolor) y 11,41 (diabetes). Dicho valor también ha sido referido por otros autores $(8,8)$, que añaden que el tipo de medicación, psicotrópicos, cardiotónicos y analgésicos están asociados a caídas ${ }^{28}$, por lo que esta puede ser una de las protagonistas de los episodios de dolor de las personas institucionalizadas.

Los resultados relativos al estado mental coinciden con lo referido por otros autores en cuanto a que las personas que padecen demencia manifiestan menos dolor'. De esta forma, en relación con el estado mental se observa que la orientación preservada, la coherencia del discurso y el comportamiento adecuado en cuanto a la orientación espacial y temporal están más asociados al riesgo de presentar dolor que los opuestos, y se manifiestan sobre todo en los que están menos deteriorados desde el punto de vista cognitivo. Esto es debido al problema de la descodificación interna y subjetiva de la sensación de dolor deficitaria en los procesos demenciales, también referido por otros autores ${ }^{12}$.

La mayoría de las personas no presenta un comportamiento adecuado y no manifiesta dolor. Algunos autores lo denominan necesidades potencialmente no satisfechas 5 . Normalmente, la alteración de estos comportamientos es interpretada como síntoma de demencia y tratada con psicotrópicos, y por lo tanto, el dolor está infratratado e infravalorado 5,25 tanto en los aspectos farmacológicos como en las medidas de confort ${ }^{15,16}$. Otros autores proponen el uso de protocolos para el tratamiento del dolor $^{29}$ y alertan del peligro de sobredosis de analgésicos en los ancianos, debido a infravalorción en cuanto a la existencia de dolor, ya que se receta el analgésico pero no se realiza una valoración permanente ${ }^{26}$. Por lo que respecta a las enfermedades psicológicas y psiquiátricas, no se han encontrado valores significativos en el estudio, aunque muchos autores refieren mayor presencia de dolor en las personas que presentan estas patologías ${ }^{14,26,29,30}$. En cuanto a la funcionalidad, el compromiso es elevado, pero la asociación con el dolor no es significativa.

\section{- CONCLUSIÓN}

Los residentes de las ULE presentan un elevado nivel de dolor. La vulnerabilidad, fragilidad, morbilidad y polimedicación relaciona- 
das con los ancianos que presentan deterioro cognitivo son factores que revelan la necesidad de que los cuidadores formales y no formales aprendan a reconocer otras expresiones de dolor además de las verbalizadas y a llevar a cabo otro tipo de actuaciones no farmacológicas que, junto con la analgesia, proporcionen mejor calidad vida a los ancianos que residen en instituciones. Asimismo, controlar el dolor y realizar protocolos de valoración y tratamiento es de suma importancia.

\section{- LIMITACIONES DEL ESTUDIO}

En primer lugar, consideramos que conocer la tasa de consumo de analgésicos y psicotrópicos sería importante para saber qué tipo de fármaco es el más consumido en una ULE.

En segundo lugar, no hacer referencia al tipo de dolor, es decir, si se trata de un dolor agudo o crónico, también nos ha parecido un factor limitante del estudio

\section{- BIBLIOGRAFÍA}

1. Achterberg WP, Gambassi G, Finne-Soveri H, Liperoti R, Noro A, Frijters DH, et al. Pain in European long-term care facilities: cross-national study in Finland, Italy and The Netherlands. Pain. 2010;148:70-4.

2. Boerlage $A A$, van Dijk M, Stronks DL, de Wit R, van der Rijt CC. Pain prevalence and characteristics in three Dutch residential homes. Eur J Pain. 2008;12:910-6

3. Takai Y, Yamamoto-Mitani N, Okamoto Y, Koyama K,Honda A Literature review of pain prevalence among older residents of nursing homes. Pain Manag Nurs. 2010;11:209-23.

4. Zwakhalen SMG, Koopmans RTCM, Geels PJEM, Berger MPF, Hamers JPH. The prevalence of pain in nursing home residents with dementia measured using an observational pain scale. Eur $J$ Pain [Internet]. European Federation of Chapters of the International Association for the Study of Pain; 2009 Jan [cited 2013 Oct 13];13(1):89-93. Disponible en: http://www.ncbi.nlm.nih.gov/ pubmed/18486509

5. Pieper MJC, van Dalen-Kok AH, Francke AL, van der Steen JT, Scherder EJ, Husebø BS, et al. Interventions targeting pain or behaviour in dementia: A systematic review. Ageing Res Rev. [Internet]. 2013 May 28 [cited 2013 Oct 6]; Disponible en: http:// www.ncbi.nlm.nih.gov/pubmed/23727161

6. Kverno KS, Rabins PV, Blass DM, Hicks KL, Black B. Prevalence and treatment of neuropsychiatric symptoms in advanced dementia. S J Gerontol Nurs. 2008:34(12):8-15.

7. Norton MJ, Allen RS, Snow AL, Hardin JM, Burgio LD. Predictors of need-driven behaviors in nursing home residents with dementia and associated certified nursing assistant burden. Aging Ment Heal. 2010;14:303-9.

8. Zuidema SU, de Jonghe JF, Verhey FR, Koopmans RT. Neuropsychiatric symptoms in nursing home patients: factor structure invariance of the Dutch nursing home version of the neuropsychiatric inventory in different stages of dementia. Dement Geriatr Cogn Disord. 2007;24:169-76.

9. Cardoso A. Manual de tratamento da dor. Lisboa: Lidel; 2013. p. 127

10. Scherder E, Herr K, Pickering G, Gibson S, Benedetti F, Lautenbacher $S$. Pain in dementia. Pain [Internet]. International Association for the Study of Pain; 2009 Oct [cited 2013 Oct 13];145(3):276-8. Disponible en: http://www.ncbi.nlm.nih.gov/pubmed/19409705

11. Correia ASA, Branco JC. Fisiopatologia da dor. 1. ${ }^{\text {a }}$ ed. Lisboa: Lidel; 2012. p. 137.

12. Kovach CR, Noonan PE, Griffie J, Muchka S, Weissman DE. 0 uso da avaliação de desconforto no protocolo de demência. Appl Enfermagem Res. 2001;14:193-200.
13. Souto P De, Lapeyre-mestre M, Vellas B, Rolland Y Potential underuse of analgesics for recognized pain in nursing home residents with dementia: A cross-sectional study. Pain [Internet] International Association for the Study of Pain; 2013:(0304):0-4. Disponible en: http://dx.doi.org/10.1016/j.pain.2013.07.017

14. Brorson H, Plymoth H, Ormon K, Bolmsjö I. Pain relief at the end of life: nurses' experiences regarding end-of-life pain relief in patients with dementia. Pain Manag Nurs. [Internet]. 2013 Feb 28 [cited 2013 0ct 6];1-9. Disponible en: http://www.ncbi.nlm.nih. gov/pubmed/23453467

15. Horgas AL, Nichols AL, Schapson C, Vietes K. Assessing pain in persons with dementia: relationships among the non-communicative patient's pain assessment instrument, self-report, and behavioral observations. Pain Manag Nurs. [Internet]. 2007 Jun [cited 2013 Oct 12];8(2):77-85. Disponible en: http://www.ncbi. nlm.nih.gov/pubmed/17544127

16. Tait RC Chibnall JT Under-treatment of pain in dementia: assessment is key. J Am Med Dir Assoc. [Internet]. 2008 Jul [cited 2013 Oct 13];9(6):372-4. Disponible en: http://www.ncbi.nlm.nih.gov/ pubmed/18585637

17. McAuliffe L, Brown DF. Pain and dementia: an overview of the literature. Int J Older People Nurs. 2012;7:219-26.

18. Ballard C, Smith J, Husebo B, Aarsland D, Corbett A. The role of pain treatment in managing the behavioural and psychological symptoms of dementia (BPSD). Int J Palliat Nurs. 2011;17:420, 422,424

19. Kverno KS, Black BS, Blass DM, Geiger-Brown J, Rabins PV Neuropsychiatric symptom patterns in hospice-eligible nursing home residents with advanced dementia. J Am Med Dir Assoc. [Internet]. 2008 Sep [cited 2013 Oct 12];9(7):509-15. Disponible en: http://www.pubmedcentral.nih.gov/articlerender.fcgi?artid= 2570193\&tool=pmcentrez\&rendertype=abstract

20. Tosato M, Lukas A, van der Roest HG, Danese P, Antocicco M, Finne-Soveri $\mathrm{H}$, et al. Association of pain with behavioral and psychiatric symptoms among nursing home residents with cognitive impairment: results from the SHELTER study. Pain [Internet]. International Association for the Study of Pain: 2012 Feb [cited 2013 0ct 13];153(2):305-10. Disponible en: http://www.ncbi.nlm. nih.gov/pubmed/22093815

21. CNAMTS. Aggir - Guide d'utilisation Paris: Securite Sociale Lássurence Maladie [Internet]. France; 2008. Disponible en: http:// vosdroits.service-public.fr/particuliers/F1229.xhtml

22. Pestana MH, Gageiro JN. Análise Categórica, Árvores de Decisão e Análise de Conteúdo em Ciências Sociais e da Saúde com 0 SPSS [Internet]. Lda, editor. Lisboa: Lidel; 2009. p. 576. Disponible en: www//Lidel edições técnicas, Lda

23. Pestana MH, Gageiro JN. Análise de Dados para Ciências Sociais: a Complementaridade do SPSS [Internet]. Lisboa; Edições Sí; 2008. p. 694. Disponible en: www//Edições Sílabo; Google: Scielo Network: ABC

24. Barry DT, Pilver CE, Hoff R, Potenza MN. Pain interference and incident mood, anxiety, and substance-use disorders: finding from a representative sample of men and women in the general population. J Psychiatr Res. [Internet]. 2013 Nov [cited 2013 Nov 26];47(11):1658-64. Disponible en: http://www.ncbi.nlm.nih.gov/ pubmed/23992771

25. De Souto Barreto P, Lapeyre-Mestre M, Vellas B, Rolland Y. Potential underuse of analgesics for recognized pain in nursing home residents with dementia: A cross-sectional study. Pain [Internet]. International Association for the Study of Pain; 2013 Nov [cited 2013 Nov 10];154(11):2427-31. Disponible en: http://www.ncbi. nlm.nih.gov/pubmed/23872102

26. De Knegt NC, Pieper MJC, Lobbezoo F, Schuengel C, Evenhuis HM, Passchier J, et al. Behavioral pain indicators in people with intellectual disabilities: a systematic review. J. Pain [lnternet] 2013 Sep [cited 2013 Nov 18];14(9):885-96. Disponible en: http:// www.ncbi.nlm.nih.gov/pubmed/23830762

27. Kapoor $P$, Kapoor A. Hypertension in the elderly: A reappraisal. Clin Queries Nephrol [Internet]. 2013 Apr [cited 2013 Nov 26];2(2):71-7. Disponible en: http://linkinghub.elsevier.com/retrieve/pii/S2211947713000149

28. Strien AM Van, Koek HL, Marum RJ Van, Emmelot-vonk $\mathrm{MH}$. Maturitas Psychotropic medications, including short acting benzodiazepines, strongly increase the frequency of falls in elderly. Maturitas [Internet]. Elsevier Ireland Ltd 2013;74(4):357-62.Disponible en: http://dx.doi.org/10.1016/j. maturitas.2013.01.004

29. Boerlage A, Valkenburg AJ, Scherder EJ, Steenhof G, Effing P Tibboel D, et al. Prevalence of pain in institutionalized adults with intellectual disabilities: a cross-sectional approach Res Dev Disabil. [Internet]. 2013 Aug [cited 2013 Oc 12];34(8):2399-406. Disponible en: http://www.ncbi.nlm.nih. gov/pubmed/23714716

30. Sotto Mayor M, Ribeiro 0, Paúl C. Satisfacción percibida en el cuidado de ancianos. Gerokomos. 2008;19(3):121-7. 